\title{
CORRESPONDENCE
}

\section{Call for an international survey on therapeutic lavage for pulmonary alveolar proteinosis}

\section{To the Editors:}

Pulmonary alveolar proteinosis (PAP) is a syndrome characterised by accumulation of proteinaceous, periodic acidSchiff positive material within airspaces due to disruption of surfactant homeostasis [1]. In $95 \%$ of cases, PAP is associated with increased serum levels of auto-antibodies neutralising granulocyte-macrophage colony-stimulating factor and, therefore, this form is referred to as "auto-immune PAP" [2].

Irrespective of the underlying cause, the current standard of care for PAP is represented by whole lung lavage (WLL) and, to a lesser extent, bronchoscopic lobar/segmental lavage [3]. WLL has played such a critical role in the management of patients affected by PAP, whose natural history had been markedly influenced by its application, that PAP is now no longer considered a potentially lethal disease. Although WLL has been performed for $50 \mathrm{yrs}$, having been first described in 1963 [4], it is still a procedure performed in selected centres that is not standardised, seldom described in detail in the scientific literature and, very often, results from a homemade apprenticeship, because of the lack of networking among specialised centres. Although widely used, WLL is still a procedure suffering from lack of information, both clinically and technically, and is far from being standardised [3].

Within the activities of the E-RARE funded European Pulmonary Alveolar Proteinosis Network (EuPAPNet) [5], and with the help of an international steering committee, we have devised a specific questionnaire for therapeutic lavage in PAP, in an attempt to fill this lack of information. The questionnaire is not limited to "lavagers" performing WLL, but also to physicians performing lobar or segmental bronchoscopic therapeutic lavages. The aim is to carry out an international survey to answer many open questions related to lavage in PAP, including the following. 1) How many centres perform lavage? 2) Where are they located? 3) How do they perform the lavage? 4) How many PAP patients are currently in follow-up in these centres?

We believe that these are very important questions for PAP management and the resulting paper(s), co-authored by all participants in the survey, will represent a real international census on PAP and how is managed, as well as being the first step in producing a consensus document on lavage in PAP.

All physicians performing therapeutic lavage in adults or children with PAP are invited to participate, even if the procedure has been implemented recently or performed in only a few patients. In an ultra-rare condition such as PAP, even the contribution of a limited sample size is critical to improve our knowledge.

The questionnaire is available from the EuPAPNet website [5] and as online supplementary material. If you are interested in participating in the survey, please ask request a copy of the questionnaire from m.luisetti@smatteo.pv.it.

We are confident that the results of the survey will represent a significant progress in the knowledge and management of PAP.

\section{Luisetti}

Correspondence: M. Luisetti, Dept Of Molecular Medicine, Section of Pneumology, University of Pavia, IRCCS San Matteo Hospital Foundation, 27100 Pavia, Italy, E-mail m.luisetti@ smatteo.pv.it

Statement of Interest: None declared.

\section{REFERENCES}

1 Whitsett JA, Wert SE, Weaver TE. Alveolar surfactant homeostasis and the pathogenesis of pulmonary disease. Annu Rev Med 2010; 61: 105-119.

2 Trapnell BC, Whitsett JA, Nakata K. Pulmonary alveolar proteinosis. N Eng J Med 2003; 349: 2527-2539.

3 Seymour JF, Preisnell JJ. Pulmonary alveolar proteinosis: progress in the first 44 years. Am J Respir Crit Care Med 2002; 166: 215-235.

4 Ramirez J, Schultz RB, Dutton RE. Pulmonary alveolar proteinosis: a new technique and a rationale for treatment. Arch Intern Med 1963; 112: 419-431.

5 EuPAPNet. European Pulmonary Alveolar Proteinosis Network. www.alveolarproteinosis.eu/index.php/en.

DOI: 10.1183/09031936.00226311 